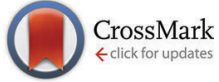

Cite this: J. Mater. Chem. C, 2016, 4, 10545

Received 28th April 2016

Accepted 18th October 2016

DOI: 10.1039/c6tc01731b

www.rsc.org/MaterialsC

\section{Elastomers with tunable dielectric and electromechanical properties $\dagger$}

\begin{abstract}
Simon J. Dünki, ${ }^{\text {ab }}$ Frank A. Nüesch ${ }^{\mathrm{ab}}$ and Dorina M. Opris*a
Novel electroresponsive silicone elastomers modified with nitrile groups are presented, whose dielectric permittivity $\left(\varepsilon^{\prime}\right)$ is tuned from $\varepsilon^{\prime}=4.3$ to $\varepsilon^{\prime}=17.4$. They are prepared in a one-step process starting from a high molecular weight poly(methylvinylsiloxane) to which polar nitrile groups and cross-links are introduced in thin films. Different ratios of butanethiol/3-mercaptoproprionitrile are used to vary the amount of nitrile groups in these elastomers, while 2,2'-(ethylenedioxy)diethanethiol is used as a crosslinker. Because of the systematic nature of this investigation, we not only present promising elastic materials with remarkable dielectric, mechanical, and electromechanical properties but also provide a guideline for materials design aimed at dielectric elastomer actuator applications.
\end{abstract}

\section{Introduction}

In the year 2000, Pelrine et al. showed that an acrylic elastomer coated on both sides with compliant electrodes is capable of producing actuation strains up to $215 \%$ under a high voltage. ${ }^{1}$ Since then, research in the dielectric elastomer actuator (DEA) field has increased significantly. Exploitation of the simple construction and operating principle of DEA has led to a plentitude of applications and a basic understanding of device concepts and materials properties. ${ }^{2-4}$ Despite the impressive number of proposed applications, very few DEAs have made it into products. ${ }^{5,6}$ The commercialization of this technology will significantly improve if dielectric elastomers were available which combine high dielectric permittivity $\left(\varepsilon^{\prime}\right)$ with excellent mechanical properties. The need for better performing materials has been recognized and is now gaining importance both in academy and industry. ${ }^{7,8}$ A high $\varepsilon^{\prime}$ material would not only reduce the operation voltage in actuators and increase the conversion of mechanical into electrical energy in generators but also increase the sensitivity of sensors. Furthermore, low voltage actuators are also of particular importance in implantable devices, where DEA could be used to replace malfunctioning muscles such as sphincters and valves. ${ }^{9}$

\footnotetext{
${ }^{a}$ Swiss Federal Laboratories for Materials Science and Technology Empa, Laboratory for Functional Polymers, Ueberlandstr. 129, CH-8600, Dübendorf, Switzerland. E-mail: dorina.opris@empa.ch

${ }^{b}$ École Polytechnique Fédérale de Lausanne (EPFL), Institut des matériaux, Station 12, CH 1015, Lausanne, Switzerland

$\dagger$ Electronic supplementary information (ESI) available: IR spectra, TGA, DSC curves, and the calculation of the Weibull parameter for $\mathbf{M}(\mathbf{1}: \mathbf{0})$, stress-strain curves at different strain rates, stress-strain curves and the actuation strain as a function of electric field for Elastosil and VHB. See DOI: 10.1039/c6tc01731b
}

The actuation voltage can be reduced by decreasing the thickness and the elastic modulus of the elastomer film, as well as by increasing its dielectric permittivity. ${ }^{10}$ Actuators with low operation voltage and high energy density would be available if high dielectric permittivity elastomers were used as a dielectric layer. Any elastic material can in principle be used as a dielectric. ${ }^{11,12}$ Silicones are however one of the most investigated materials in the DEA field. ${ }^{13}$ They are easy to process in thin films with excellent mechanical and dielectric properties. They have however a rather low $\varepsilon^{\prime}$ of about 3. Attempts to increase their permittivity rely on either blending with highly polarizable organic or inorganic fillers or chemical modification with polar groups. ${ }^{14}$ Significant progress has been achieved in regard to polar silicones with enhanced permittivity. ${ }^{4}$ Unfortunately, only very few polar silicones have been investigated in electromechanical tests. One common problem faced is that these polar silicones show only a moderate increase in permittivity ${ }^{15,16}$ or meet only partially the requirements for actuators, and therefore are often used as high permittivity fillers in a silicone matrix with excellent mechanical properties. ${ }^{17-20}$ It would be an obvious advantage if blending could be obviated and the cross-linked polar silicones themselves would have attractive mechanical properties and high permittivity.

We have recently shown that it is possible to prepare high molar mass polysiloxanes containing different mol\% of polar CN side groups. ${ }^{21,22}$ The permittivity of such highly viscous polymers was tuned from 4.7 to 18.4. Several attempts from our laboratory to combine such attractively high permittivity with good elastic properties (large and reversible elongations) failed during the cross-linking step. In a first attempt to overcome this problem, a one-step process to prepare silicone elastomers was developed which allowed the synthesis of a material with a permittivity of $\varepsilon^{\prime}=10.1$ at high frequencies. ${ }^{23}$ Even though this 
achievement is substantial, the chemical structures and the concept involved suggest that a permittivity of approximately 18 would be within reach. The likely reason for the obvious failure to reach this high value is seen in the photo-curing step to which thin films of this material are subjected to. The films are prepared on a substrate leaving their upper side exposed to air. If such films are now irradiated in order to bring about crosslinking and nitrile addition, the volatile thiol may evaporate leaving some vinyl groups unreacted, which then causes the permittivity to be lower than anticipated. IR investigations of the film surface exposed to air confirmed the presence of unreacted vinyl groups.

Here, we report on a novel polysiloxane which not only combines a permittivity of 17.4 with excellent mechanical properties but also does not require blending with other materials anymore. Thus, this novel polysiloxane recommends itself for direct use as the final material in DEA devices. Furthermore, we describe a way to systematically tune the permittivity in the range of 4.3-17.4 while maintaining the same cross-link density in the elastomers. This provides a unique insight into how the nitrile content impacts critical materials properties such as permittivity, dielectric loss, conductivity, and elastic modulus. Since all these properties have an impact on the electromechanical characteristics we believe that our findings are an important basis for rational materials design.

\section{Experimental}

\subsection{Materials}

Unless otherwise stated, all chemicals were of reagent grade and used without purification. 1,3,5,7-Tetramethyl-1,3,5,7tetravinyl cyclotetrasiloxane $\left(\mathrm{V}_{4}\right)$ was purchased from ABCR. 2,2-Dimethoxy-2-phenylacetophenone (DMPA), 2,2'-(ethylenedioxy)diethanethiol, benzene, toluene, 3-chloropropionitrile, tetramethylammonium hydroxide $25 \%$ in $\mathrm{MeOH}$ (TMAH), and thiourea were purchased from Aldrich. Amorphous $\mathrm{SiO}_{2}$ particles (60-70 nm) were purchased from US Research Nanomaterials Inc., Houston. Methanol, dichloromethane, hexane, ethyl acetate, and tetrahydrofuran were purchased from VWR and 1,1,1,3,3,3hexamethyldisilazane was obtained from Alfa Aesar. Elastosil RT 745 "S" A+B (Wacker) was purchased from Drawin Vertriebs GmbH. VHB 4905 was kindly provided by Mr M. Silvain (Empa). Thin films of Elastosil were prepared according to the method reported by Dünki et al. ${ }^{23}$

\subsection{Characterization}

${ }^{1} \mathrm{H}$ - and ${ }^{13} \mathrm{C}-\mathrm{NMR}$ spectra were recorded at $298 \mathrm{~K}$ on a Bruker Avance 400 NMR spectrometer using a $5 \mathrm{~mm}$ broadband inverse probe at 400.13 and $100.61 \mathrm{MHz}$, respectively. Chemical shifts $(\delta)$ in ppm were calibrated to residual solvent peaks $\left(\mathrm{CDCl}_{3}\right.$ : $\delta=7.26$ and $77.16 \mathrm{ppm}, \mathrm{D}_{2} \mathrm{O} \delta=4.79$ ). Size exclusion chromatograms were recorded using an Agilent 1100 Series HPLC (Columns: serial coupled PSS SDV $5 \mu, 100 \mathrm{~A}$ and PSS SDV $5 \mu$, 1000A, detector: DAD, $235 \mathrm{~nm}$ and $360 \mathrm{~nm}$; refractive index), with THF as mobile phase. PDMS standards were used for the calibration and toluene as an internal standard. Swelling-extraction tests were conducted by immersing the samples in THF at room temperature for 5 days. Every $24 \mathrm{~h}$ the solvent was replaced. The films were dried to constant mass at $50{ }^{\circ} \mathrm{C}$ under reduced pressure. IR spectra were recorded on a Bruker Tensor 27 FT-IR using either an ATR setup or as $\mathrm{KBr}$ pellet. The tensile tests were performed on a Zwick Z010 tensile test machine with a crosshead speed of $50 \mathrm{~mm} \min ^{-1}\left(278 \% \mathrm{~min}^{-1}\right)$. Tensile test specimens with a gauge width of $2 \mathrm{~mm}$ and a gauge length of $18 \mathrm{~mm}$ were prepared by die cutting. The strain was determined using a longitudinal strain extensometer. The curves were averaged from 3 different samples per material (see ESI $\dagger$ ). The tensile modulus was determined from the slope of the stress-strain curves using a linear fit to the data points within 10\% strain. Crosslink density was determined according to the literature. ${ }^{24,25}$ The averaged tensile test values were used for the calculation. The intercept $C_{1}$ of a linear fit for $1 / \lambda$ values between 0.8 and 0.55 in the $\sigma /\left(\lambda-\lambda^{-2}\right)$ vs. $1 / \lambda$ plot was determined. The crosslinking density was calculated as

$$
\rho_{\mathrm{CL}}=\frac{C_{1}}{R T},
$$

where $R$ is the gas constant and $T$ the temperature. Dynamic mechanical analysis was carried out on a RSA 3 DMA from TA Instruments. Stripes of $10 \mathrm{~mm} \times 20 \mathrm{~mm}$ were measured under a dynamic load of $2.5 \mathrm{~g}$, at $2 \%$ strain in the frequency range of $0.05-10 \mathrm{~Hz}$ at $25{ }^{\circ} \mathrm{C}$ and $65 \%$ humidity. Permittivity measurements were performed in the frequency range from $1 \mathrm{~Hz}$ to $1 \mathrm{MHz}$ using a Novocontrol Alpha-A frequency analyzer. The $V_{\mathrm{RMS}}$ (root mean square voltage) of the probing AC electric signal applied to the samples was $1 \mathrm{~V}$. The samples were squeezed between two electrodes (diameter of $5 \mathrm{~mm}$ ). The electromechanical tests were performed using circular membrane actuators, for which the films were fixed between two circular frames. A biaxial prestrain of $28.6 \%$ was used. Circular electrodes ( $8 \mathrm{~mm}$ diameter) of carbon black powder were applied to each side of the film. A FUG HCL35-12500 high voltage source served as power supply for actuator tests. The voltage was increased by $50 \mathrm{~V}$ steps every $2 \mathrm{~s}$ up to breakdown. The actuation strain was measured optically as the extension of the diameter of the electrode area via a digital camera, using an edge detection tool of a LabView program to detect the boundary between the black electrode area and the transparent silicone film. The dielectric breakdown field $\left(E_{\mathrm{b}}\right)$ of the films was measured by placing the films between two flat electrodes with a $1 \mathrm{~mm}^{2}$ area which were embedded in an epoxy resin. The voltage was gradually increased until the breakdown occurred.

\subsection{Synthesis of 2-cyanoethylthiouronium hydrochloride}

To a solution of thiourea (57.5 g, $0.755 \mathrm{~mol}, 1.37$ equiv.) in water (38 ml), 3-chloropropionitrile ( $49.3 \mathrm{~g}, 0.551 \mathrm{~mol}, 1$ equiv.) was added and heated to $100{ }^{\circ} \mathrm{C}$ for $2 \mathrm{~h}$. The reaction mixture was cooled in an ice/salt bath to crystallize the product. The precipitate was dispersed in acetone $(160 \mathrm{ml})$ and then filtered. The filter cake was washed with cold acetone and ether and dried under reduced pressure to yield 2-cyanoethylthiouronium hydrochloride as white crystals (78.9 g, $0.476 \mathrm{~mol}, 86 \%$ ). 
${ }^{1} \mathrm{H}$ NMR $\left(400 \mathrm{MHz}, \mathrm{D}_{2} \mathrm{O}, \delta\right): 3.47\left(\mathrm{t}, J=6.72 \mathrm{~Hz}, 2 \mathrm{H}, \mathrm{S}-\mathrm{CH}_{2}\right) ; 3.02$ (t, $\left.J=6.72 \mathrm{~Hz}, 2 \mathrm{H}, \mathrm{CH}_{2}-\mathrm{CN}\right)$.

\subsection{Synthesis of 1-mercaptopropionitrile $(2)^{26}$}

An aq. $\mathrm{NaOH}$ sol. (11.2 M, $78 \mathrm{ml}, 0.874 \mathrm{~mol}, 1.8$ equiv.) was added dropwise to a solution of 2-cyanoethylthiouronium hydrochloride ( $78.9 \mathrm{~g}, 0.476 \mathrm{~mol}, 1$ equiv.) in water $(210 \mathrm{ml})$ under $\mathrm{Ar}$ at $15-25{ }^{\circ} \mathrm{C}$. It was then heated to $45{ }^{\circ} \mathrm{C}$ for an additional $1 \mathrm{~h}$. The reaction mixture was cooled in an ice/salt bath, $\mathrm{H}_{2} \mathrm{SO}_{4}(\mathrm{ca} .14 \mathrm{ml}$ ) was added to adjust the $\mathrm{pH}$ to 6 , and the solution was then extracted with diethyl ether $(6 \times 100 \mathrm{ml})$. The combined organic phases were dried over $\mathrm{MgSO}_{4}$, filtrated, and the solvent was removed. The reaction mixture was distilled to give a colourless liquid (19.6 g, $0.225 \mathrm{~mol}, 47 \%) .{ }^{1} \mathrm{H}$ NMR $\left(400 \mathrm{MHz}, \mathrm{CDCl}_{3}, \delta\right): 2.82-2.76\left(\mathrm{~m}, 2 \mathrm{H}, \mathrm{S}-\mathrm{CH}_{2}\right) ; 2.71-2.67$ (m, $\left.2 \mathrm{H}, \mathrm{CH}_{2}-\mathrm{CN}\right) ; 1.80(\mathrm{t}, J=8.5,1 \mathrm{H})$.

\subsection{Synthesis of materials $M(x: y)$}

A suspension of functionalized $\mathrm{SiO}_{2}$ nanoparticles $(231 \mathrm{mg})$ in THF $(10 \mathrm{ml})$ was sonicated for $5 \mathrm{~min}$ using an ultrasonicator tip. To this, poly(methylvinylsiloxane) ( $2.00 \mathrm{~g}, 23.2 \mathrm{mmol}, 1$ equiv.) was added and stirred until it dissolved. Thiols 1 (1.01 g, $11.2 \mathrm{mmol}, 0.48$ equiv.), 2 ( $0.98 \mathrm{~g}, 11.2 \mathrm{mmol}, 0.48$ equiv.) and 3 ( $28 \mu \mathrm{l}, 0.17 \mathrm{mmol}, 0.007$ equiv.), and DMPA (14 mg, $0.05 \mathrm{mmol}$, 0.002 equiv.) were added and the mixture was stirred to form a homogenous solution. The reaction mixture was poured on a Teflon substrate and covered with a glass plate, whereby two $300 \mu \mathrm{m}$ spacers were used to adjust the thickness of the film. The film formed after UV-irradiation for 4 min was dried at $50{ }^{\circ} \mathrm{C}$ for $24 \mathrm{~h}$.

\section{Results and discussion}

A series of silicone elastomers containing polar CN pendant groups was prepared in a one-step process as shown in Scheme 1. To ensure good mechanical properties for the cross-linked materials and a maximum degree of modification with CN groups, a high molecular weight polysiloxane $\mathbf{P}$ that contains vinyl groups at every siloxy repeat unit was used as the starting material. Polymer $\mathbf{P}$ was synthesized in a $200 \mathrm{~g}$ batch by anionic polymerization of 1,3,5,7-tetramethyl-1,3,5,7-tetravinylcyclotetrasiloxane $\left(\mathrm{V}_{4}\right)$ in the presence of tetramethylammonium hydroxide (TMAH) following standard procedures and was used

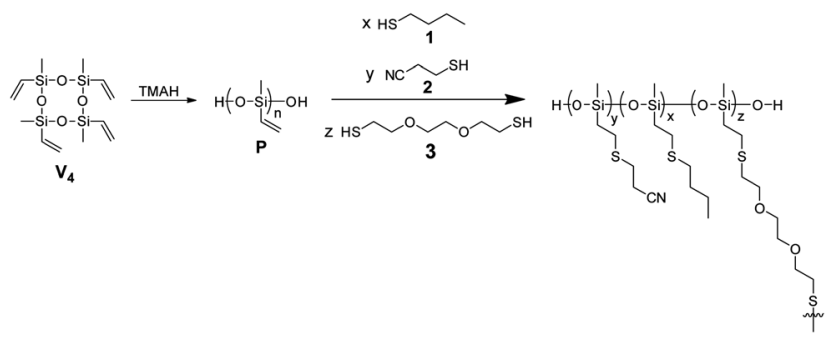

Scheme 1 Synthesis of poly(methylvinylsiloxane) $\mathbf{P}$ from $V_{4}$ monomer and in situ functionalization and cross-linking of $\mathbf{P}$ with butanethiol/ 3-mercaptopropionitrile and 2,2' - (ethylenedioxy)diethanethiol, respectively, via an UV-induced thiol-ene reaction. as the stock for subsequent materials formation. The molecular weight and its distribution $\left(M_{\mathrm{n}}=85 \mathrm{~kg} \mathrm{~mol}^{-1}, M_{\mathrm{w}}=209 \mathrm{~kg} \mathrm{~mol}^{-1}\right.$, and $D=2.46$ ) were determined by GPC in tetrahydrofuran (THF) using poly(dimethylsiloxane) standards. The vinyl groups of polysiloxane $\mathbf{P}$ served two purposes: attaching polar $\mathrm{CN}$ groups and cross-linking two polysiloxane chains via a bifunctional thiol. For the modification of $\mathbf{P}$ with polar groups, a wide range of polar thiols can in principle be used. We selected 3-mercaptopropionitrile (2) because it is easily accessible in large quantities from rather cheap materials and it contains polar $\mathrm{CN}$ groups which are easy to polarize by an electric field.

To tune the amount of $\mathrm{CN}$ groups, $\mathbf{P}$ was reacted with 1-butanethiol (1) and 2 separately and in various ratios $x: y$, as well as with the dithiol 3 cross-linker so as to create materials $\mathbf{M}(\boldsymbol{x}: \boldsymbol{y})$ with different contents of $\mathrm{CN}$ groups $(y)$. The amount of cross-linker 3 used has a direct impact on the mechanical properties of $\mathbf{M}(\boldsymbol{x}: \boldsymbol{y})$. In a typical experiment a solution of $\mathbf{P}$, an approximately equimolar amount of functional thiols $(\mathbf{1}, 2$ or a mixture of $1: 2$ ), the selected amount of dithiol cross-linker 3, and DMPA were placed between a glass plate and a Teflon substrate. The distance between the plates was adjusted by spacers. During UV-irradiation, the dipole attachment and the cross-linking in thin films occur simultaneously. The top glass plate was immediately removed after irradiation, and the films were carefully peeled off from the glass plate (Fig. 1). All films yielded elastic materials upon cross-linking.

Since we wanted to keep the amount of cross-linker constant for all materials, the optimum concentration that allowed the formation of elastomers irrespective of the $\mathrm{CN}$ group content had to be found. This concentration was optimized for the most challenging material, which turned out to be the one which had only butyl thioether groups $\mathbf{M}(\mathbf{1 : 0})$. As mentioned in the Introduction, the synthesis of a material fully functionalized with $\mathrm{CN}$ groups was recently described by us, and therefore the reported recipe was used as the starting point for our investigations. When material $\mathbf{M}(\mathbf{1}: \mathbf{0})$ was prepared using the reported recipe, except that thiol 2 was replaced by $\mathbf{1}$, a rather brittle material with low strain at break formed. Therefore, we gradually decreased the amount of $\mathbf{3}$ from $23 \mu \mathrm{l}$ to $10 \mu \mathrm{l}$ per $\mathrm{g}$ of $\mathbf{P}$. The stress-strain curves of the resulting materials are shown in Fig. 2, while the amounts of reagents used are given in Table 1. All materials showed a strain at break of more than $100 \%$. As expected, the materials turned softer with the decreasing amount of cross-linker since the cross-link density is decreased. The best material of composition $\mathbf{M}(\mathbf{1}: \mathbf{0})$

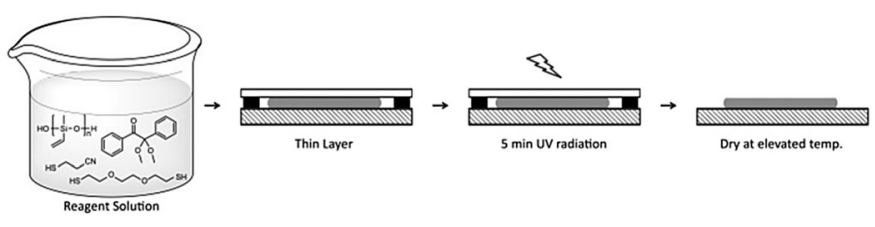

Fig. 1 A one-step process to prepare silicone elastomer films with tuneable content of $\mathrm{CN}$ groups. A solution of $\mathbf{P}, 2,2^{\prime}$-(ethylenedioxy)diethanethiol cross-linker, different ratios of functional thiols (butanethiol:3-mercaptopropionitrile), and DMPA UV-initiator in THF was placed between two glass plates and UV-irradiated to form elastic films. 


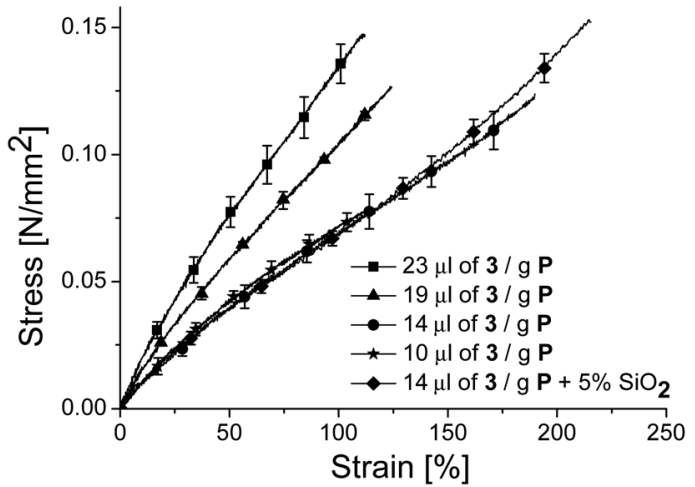

Fig. 2 Stress-strain curves of material $\mathbf{M}(\mathbf{1}: \mathbf{0})$ for which the amount of bifunctional thiol cross-linker $\mathbf{3}$ was tuned. Each curve is the average of three samples (see ESI†).

Table 1 Amounts of reagents used for the synthesis of materials $M(x: y)$

\begin{tabular}{llllllll}
\hline Name & $\mathbf{P}[\mathrm{g}]$ & $\mathbf{1}[\mathrm{g}]$ & $\mathbf{2}[\mathrm{g}]$ & $\mathbf{3}[\mu \mathrm{l}]$ & $\mathrm{SiO}_{2}[\mathrm{wt} \%]$ & $\mathrm{THF}[\mathrm{ml}]$ & Spacer $\mu \mathrm{m}$ \\
\hline $\mathbf{M}(\mathbf{1 : 0 )}$ & 1 & 1.00 & - & 23 & - & 4 & 300 \\
$\mathbf{M}(\mathbf{1 : 0 )}$ & 1 & 1.00 & - & 19 & - & 4 & 300 \\
$\mathbf{M}(\mathbf{1 : 0 )}$ & 1 & 1.00 & - & 14 & - & 4 & 300 \\
$\mathbf{M}(\mathbf{1 : 0 )}$ & 1 & 1.00 & - & 10 & - & 4 & 300 \\
$\mathbf{M}(\mathbf{1 : 0 )}$ & 1 & 1.00 & - & 14 & 4.8 & 5 & 300 \\
$\mathbf{M}(\mathbf{3 : 1 )}$ & 2 & 1.52 & 0.49 & 28 & 4.8 & 10 & 300 \\
$\mathbf{M}(\mathbf{1 : 1 )}$ & 2 & 1.01 & 0.98 & 28 & 4.8 & 10 & 300 \\
$\mathbf{M}(\mathbf{1 : 3 )}$ & 2 & 0.50 & 1.45 & 28 & 4.8 & 10 & 300 \\
$\mathbf{M}(\mathbf{0}: \mathbf{1})$ & 2 & - & 1.96 & 28 & 4.8 & 10 & 300
\end{tabular}

had a strain at break of $190 \%$ and a tensile strength of $122 \mathrm{kPa}$ (Fig. 2). A small improvement in the mechanical properties of $\mathbf{M}(\mathbf{1}: \mathbf{0})$ was achieved when $5 \mathrm{wt} \%$ of hexamethyldisilazane treated silica particles were used.

For the synthesis of materials $\mathbf{M}(\boldsymbol{x}: \boldsymbol{y})$ the amount of $\mathbf{3}$ was kept constant (14 $\mu$ l per g P) (Table 1) and a dispersion of hexamethyldisilazane treated silica particles in THF was used, as it is known that silica particles can reinforce the silicones and increase their strain at break. The amount of silica was kept below $5 \%$ so that the elastic moduli are not much affected.

The conversion of the vinyl groups in the films was investigated by FT-IR measurements conducted on the two surfaces as well as with the pellets obtained by pressing the powders of ground films in $\mathrm{KBr}$. The spectra were normalized to the highest peak assigned to the $\mathrm{Si}-\mathrm{O}-\mathrm{Si}$ st vibration at $1025 \mathrm{~cm}^{-1}$. For the vinyl group three characteristic signals at $\nu=3055 \mathrm{~cm}^{-1}, 3020 \mathrm{~cm}^{-1}$ and $2965 \mathrm{~cm}^{-1}$ with the one at $3055 \mathrm{~cm}^{-1}$ having the highest intensity can be easily identified. The signal at $\nu=2250 \mathrm{~cm}^{-1}$ is characteristic of the $\mathrm{CN}$ groups. With the increasing dipole content, the intensity of the $\mathrm{C} \equiv \mathrm{N}$ st band at $2250 \mathrm{~cm}^{-1}$ increased, while that of the $\mathrm{C}-\mathrm{H}$ st bands at 2956 and $2929 \mathrm{~cm}^{-1}$ decreased. When covering the thin film with a glass plate during irradiation, the IR spectra clearly showed an elevated conversion of the vinyl groups. The IR spectra of the pellets obtained by pressing the powders of ground films in $\mathrm{KBr}$ show the presence of less than $4 \%$ unreacted vinyl groups. Some residual vinyl groups were expected, since a slightly less than stoichiometric amount of thiols to vinyl groups was used. However, as compared with our previous report ${ }^{23}$ where the film surface exposed to air had most of the vinyl groups unreacted, here, the top surface covered by the glass plate had most of the vinyl groups reacted (Fig. S1, ESI $\dagger$ ).

The densities of $\mathbf{M}(\boldsymbol{x}: \boldsymbol{y})$ were measured via hydrostatic weighting in ethanol. The materials get denser with the increasing content of nitrile groups from $1.084 \mathrm{~g} \mathrm{~cm}^{-3}$ for $\mathbf{M}(\mathbf{1}: \mathbf{0})$, to $1.118 \mathrm{~g} \mathrm{~cm}^{-3}$ for $\mathbf{M}(\mathbf{1}: 1)$, and reached a maximum value of $1.180 \mathrm{~g} \mathrm{~cm}^{-3}$ for $\mathbf{M}(\mathbf{0}: \mathbf{1})$.

The swelling-extraction tests show that all materials contain about $10 \%$ extractable species, except for $\mathbf{M}(\mathbf{1}: \mathbf{0})$ for which the amount of extractable species was $13.4 \%$. The crosslink density was determined by the Mooney-Rivlin equation using the data obtained from the stress-strain experiments. In principle, the Flory-Rhener model could also be used, if the Flory-Huggins interaction parameter of materials $\mathbf{M}(\boldsymbol{x}: \boldsymbol{y})$ were known. It was found that $\mathbf{M}(\mathbf{1}: \mathbf{0})$ has the lowest cross-link density of $18 \mathrm{~mol} \mathrm{~m}^{-3}$, which explains the slightly higher extraction fraction observed for this material, while the cross-link density for all other materials was between 21 and $26 \mathrm{~mol} \mathrm{~m}^{-3}$ (Table 2).

The thermal stability of $\mathbf{M}(\boldsymbol{x}: \boldsymbol{y})$ was investigated by TGA (Fig. S2, ESI $\dagger$ ). All materials are stable up to $160{ }^{\circ} \mathrm{C}$ where a small amount of volatiles is removed, while above $300{ }^{\circ} \mathrm{C}$ the materials lose more than half of their weight. Differential scanning calorimetry (DSC) measurements show an increase in $T_{\mathrm{g}}$ with the increasing mol\% of nitrile groups from $-92.6{ }^{\circ} \mathrm{C}$ for $\mathbf{M}(1: 0)$ to $-67.7{ }^{\circ} \mathrm{C}$ for $\mathbf{M}(\mathbf{1}: \mathbf{1})$, and reached a maximum value of $-49.2{ }^{\circ} \mathrm{C}$ for $\mathbf{M}(0: 1)$ (Fig. 3 and Fig. S3, ESI $\dagger$ ). No melting points and no other transitions were observed. Table 3 summarizes the transition temperatures observed for $\mathbf{M}(\boldsymbol{x}: \boldsymbol{y})$ as well as the $\Delta C_{\mathrm{p}}$ of the transitions. Despite the shift of $T_{\mathrm{g}}$ towards higher values due to the chemical modification of $\mathbf{P}$ with CN groups, the $T_{\mathrm{g}}$ of all materials was well below room temperature. The increase in $T_{\mathrm{g}}$ and $\Delta C_{\mathrm{p}}$ with the $\mathrm{CN}$ content suggests the presence of some dipole-dipole interactions which restrict the mobility of the polysiloxane segments.

Tensile and DMA investigations were conducted on all materials $\mathbf{M}(\boldsymbol{x}: \boldsymbol{y})$. Fig. 4 shows the stress-strain curves of different materials. It can be seen that the elastomers get stiffer with the increasing amount of $\mathrm{CN}$ groups incorporated and the strain at break and the tensile strength increase. All materials were prepared using the same batch of starting polymer and the same amount of cross-linker, the only parameter changed was the ratio of thiols $\mathbf{1}$ and 2 . Therefore, the change in the

Table 2 Density, extraction loss, and cross-link density as calculated by the Mooney-Rivlin equation and by using the amount of reagent $3(14 \mu \mathrm{l}$ per $\mathrm{g}$ P) for different materials $\mathbf{M}(\boldsymbol{x}: \boldsymbol{y})$

\begin{tabular}{llllll}
\hline Sample & $\mathbf{M}(\mathbf{1}: \mathbf{0})$ & $\mathbf{M}(\mathbf{3}: \mathbf{1})$ & $\mathbf{M}(\mathbf{1}: \mathbf{1})$ & $\mathbf{M}(\mathbf{1}: \mathbf{3})$ & $\mathbf{M}(\mathbf{0}: \mathbf{1})$ \\
\hline Density $\left[\mathrm{g} \mathrm{cm}^{-3}\right]$ & 1.084 & 1.097 & 1.118 & 1.154 & 1.180 \\
Extraction loss [\%] & 13.4 & 10.4 & 8.8 & 10.0 & 11.1 \\
XL density $^{a}$ & 18 & 24 & 25 & 21 & 26 \\
XL density $^{b}$ & 63 & 63 & 65 & 67 & 68
\end{tabular}

${ }^{a}$ Calculated using the Mooney-Rivlin equation $\left[\mathrm{mol} \mathrm{m}^{-3}\right] .{ }^{b}$ Calculated using the amount of starting materials $\left[\mathrm{mol} \mathrm{m}^{-3}\right]$. 


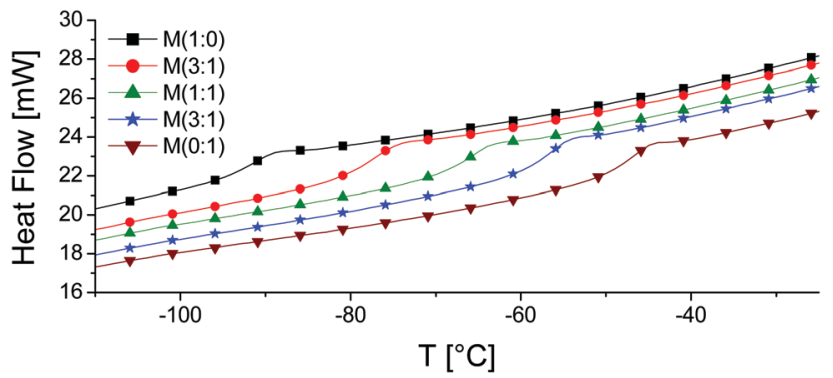

Fig. 3 DSC curves of $M(x: y)$. An increase in $T_{g}$ with the $C N$ content can be seen.

Table 3 Glass transition temperatures observed for $\mathbf{M}(\boldsymbol{x}: \boldsymbol{y})$ as well as the $\Delta C_{p}$ of the transitions

\begin{tabular}{|c|c|c|c|c|}
\hline \multirow[b]{2}{*}{ Material } & \multicolumn{2}{|l|}{$\underline{T_{\mathrm{g}}\left[{ }^{\circ} \mathrm{C}\right]}$} & \multicolumn{2}{|c|}{$\underline{\Delta C_{\mathrm{p}}\left[\mathrm{J} \mathrm{g}^{-1}{ }^{\circ} \mathrm{C}^{-1}\right]}$} \\
\hline & 2nd Heating & Cooling & 2nd Heating & Cooling \\
\hline$M(1: 0)$ & -92.6 & -102.3 & 0.30 & 0.31 \\
\hline$M(3: 1)$ & -78.3 & -88.3 & 0.35 & 0.37 \\
\hline$M(1: 1)$ & -67.7 & -75.2 & 0.38 & 0.46 \\
\hline$M(1: 3)$ & -58.2 & -65.5 & 0.44 & 0.43 \\
\hline$M(0: 1)$ & -49.2 & -56.5 & 0.44 & 0.45 \\
\hline
\end{tabular}

mechanical properties of $\mathbf{M}(\boldsymbol{x}: \boldsymbol{y})$ can be attributed to the presence of $\mathrm{CN}$ groups. The reason behind this is the dipoledipole interactions which slightly hinder the slipping of the segments, and therefore more stress is needed to deform the materials. The hindered freedom of segment motion is reflected by the increase in the $T_{\mathrm{g}}$ and $\Delta C_{\mathrm{p}}$ and also reported in the literature. ${ }^{27,28}$ Sample $\mathbf{M}(\mathbf{1}: \mathbf{0})$ is the softest very likely due to its lower cross-link density and the plasticising effect of butyl groups. Because $\mathbf{M}(\mathbf{1}: \mathbf{0})$ does not contain $\mathrm{CN}$ groups large segments can move over each other under a mechanical stress. The elastic moduli at different strain levels are summarised in Table 4. The moduli increase from $89 \mathrm{kPa}$ for $\mathbf{M}(\mathbf{1}: \mathbf{0})$ to $97 \mathrm{kPa}$ for $\mathbf{M}(1: 1)$ and reach a maximum value of $155 \mathrm{kPa}$ for $\mathbf{M}(\mathbf{0}: \mathbf{1})$. The strain at break of the films rose from about $210 \%$ for $\mathbf{M}(\mathbf{1}: \mathbf{0})$ to $290 \%$ for $\mathbf{M}(\mathbf{1}: \mathbf{1})$ up to about $320 \%$ for $\mathbf{M}(\mathbf{0}: \mathbf{1})$. The

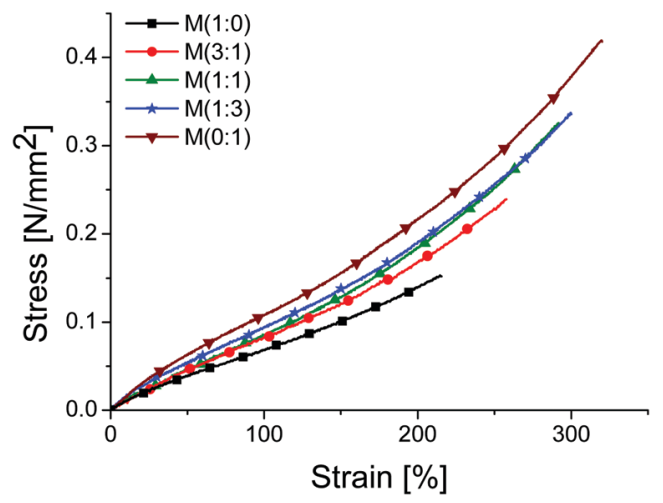

Fig. 4 Stress-strain curves for materials $M(x: y)$ from standard tensile tests at $50 \mathrm{~mm} \mathrm{~min}^{-1}$. The stress-strain curves were averaged from three independent tests.
Table 4 Storage moduli $E^{\prime}$ at $0.05 \mathrm{~Hz}$ and elastic moduli at $10 \%$ strain $\left(Y_{10 \%}\right)$ for $M(x: y)$

\begin{tabular}{llllll}
\hline Sample & $\mathbf{M}(\mathbf{1}: \mathbf{0})$ & $\mathbf{M}(\mathbf{3}: \mathbf{1})$ & $\mathbf{M}(\mathbf{1 : 1})$ & $\mathbf{M}(\mathbf{1}: \mathbf{3})$ & $\mathbf{M}(\mathbf{0}: \mathbf{1})$ \\
\hline$E^{\prime a}[\mathrm{kPa}]$ & 94 & 104 & 105 & 103 & 111 \\
$Y_{10 \%}[\mathrm{kPa}]$ & 89 & 96 & 97 & 128 & 155 \\
${ }^{a}$ Taken at $0.05 \mathrm{~Hz}$. & & & & \\
\hline
\end{tabular}

positive increase in the strain at break observed for $\mathbf{M}(\mathbf{0 : 1 )}$ is mostly due to the dipole-dipole interactions which hinder to some extent the crack propagation. ${ }^{29}$

Stress-strain curves obtained from standard tensile tests at various initial strain rates $\left(0.463 \mathrm{~s}^{-1}, 0.0463 \mathrm{~s}^{-1}, 0.00463 \mathrm{~s}^{-1}\right.$, and $0.00463 \mathrm{~s}^{-1}$ ) overlapped and an increase in the strain at break with the increasing strain rate was observed for all materials (see ESI $\dagger$ ). The increased strain at break at higher initial strain rates might be related to increased creeping in the materials at higher rates. To elucidate this, dynamic mechanical frequency sweep measurements between 0.05 and $10 \mathrm{~Hz}$ for $\mathbf{M}(\boldsymbol{x}: \boldsymbol{y})$ were also conducted (Fig. 5). The measurements turned noisier above $3 \mathrm{~Hz}$, indicating that the elastomer cannot follow the modulated amplitude. Nevertheless, the max $\tan (\delta)$ values at about $6 \mathrm{~Hz}$ were below 0.4 , a sign that the materials did not yet reach the viscoelastic regime and are still elastic. For all materials, the storage moduli $E^{\prime}$ at $0.05 \mathrm{~Hz}$ range between $100 \mathrm{kPa}$ and $110 \mathrm{kPa}$ and they only slightly increase with an elevated content of nitrile groups. $\mathbf{M}(\mathbf{1}: \mathbf{0})$ shows only a minor dependence of $E^{\prime}$ and $\tan (\delta)$ on the strain rate, while $\mathbf{M}(0: 1)$ shows a significant dependence of $E^{\prime}$ on the strain rate. For example, an increase in $E^{\prime}$ from $110 \mathrm{kPa}$ at $0.05 \mathrm{~Hz}$ to $195 \mathrm{kPa}$ at $5 \mathrm{~Hz}$ was observed for material $\mathbf{M}(\mathbf{0}: \mathbf{1})$ that has the highest content of nitrile groups. An increase of $\tan (\delta)$ with frequency and with the increasing content of nitrile groups was noted. For example, the $\tan (\delta)$ of $\mathbf{M}(\mathbf{0}: \mathbf{1})$ changed from 0.08 at $0.05 \mathrm{~Hz}$ to 0.39 at $5 \mathrm{~Hz}$. The storage modulus at low frequencies

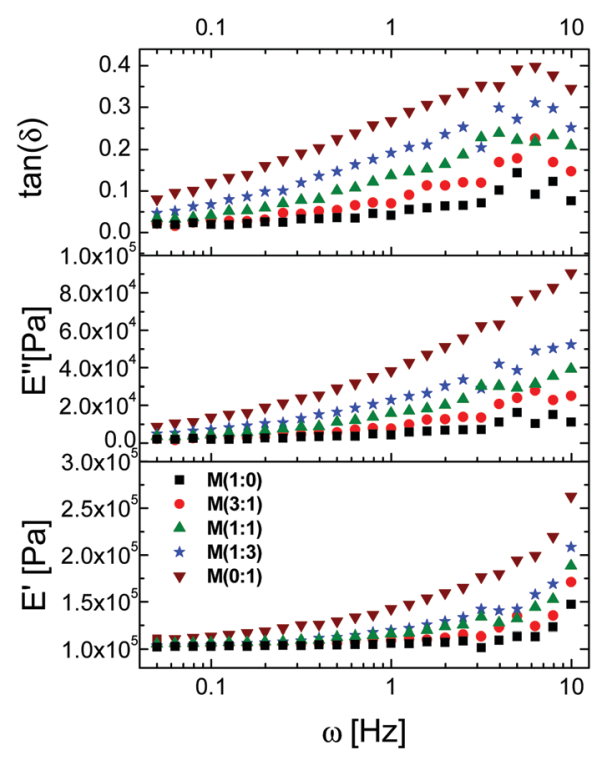

Fig. 5 Dynamic mechanical analysis for $\mathbf{M}(x: y)$ at different frequencies. 
and the Young's modulus at low strains from the tensile test are quite similar (Table 4).

The permittivity of $\mathbf{M}(\boldsymbol{x}: \boldsymbol{y})$ was tuned by reacting different ratios of mercaptopropionitrile/butanethiol with $\mathbf{P}$. As expected, a linear increase in permittivity $\varepsilon^{\prime}$ with the increasing content of nitrile groups was observed. The $\varepsilon^{\prime}$ values at high frequencies increased from 4.3 for $\mathbf{M}(\mathbf{1}: \mathbf{0})$ via 9.7 for $\mathbf{M}(\mathbf{1}: \mathbf{1})$ to the maximum of 17.4 for $\mathbf{M}(\mathbf{0}: \mathbf{1})$, which has a $\mathrm{CN}$ group at every repeat unit (Fig. 6).

The improved process to form thin films has a direct influence on their dielectric properties. For example, when we aimed at elastomers with the maximum content of CN groups, $\mathbf{M}(\mathbf{0}: \mathbf{1})$, the film where one surface was exposed to air showed a permittivity value of 10.1 at $10 \mathrm{kHz}$ and a relaxation peak at $500 \mathrm{~Hz}$ that resulted in a further increase in permittivity, ${ }^{23}$ while the film cured by the optimized method allowed the formation of $\mathbf{M}(\mathbf{0}: 1)$ with permittivity as high as $\varepsilon^{\prime}=17.4$ at $10 \mathrm{kHz} .{ }^{30}$ A surge in permittivity at low frequencies for all materials modified with CN groups was observed. This increase is very likely due to electrode polarization, when ion impurities accumulate at the electrode. This effect is higher for the materials that have a high content of $\mathrm{CN}$ groups and is shifted towards higher frequencies. Unfortunately, the dielectric losses $\varepsilon^{\prime \prime}$ increase at higher dipole contents. A similar behaviour was observed for the conductivity $\sigma$. For example, material $\mathbf{M}(\mathbf{1}: \mathbf{0})$ has a $\sigma=3.5 \times 10^{-11} \mathrm{~S} \mathrm{~cm}^{-1}$ at $10 \mathrm{kHz}$, while $\mathbf{M}(0: 1)$ reached a value of $\sigma=6 \times 10^{-9} \mathrm{~S} \mathrm{~cm}^{-1}$, values that are still typical for insulators. The increase in conductivity with the CN content is mainly due to increased ion mobility which is favoured by the presence of CN groups. It is still unclear how the materials got contaminated with ions and if it is possible to avoid them by using ultra-pure reagents, solvents, and glassware. Further work is required to elucidate this aspect.

All materials $\mathbf{M}(\boldsymbol{x}: \boldsymbol{y})$ as well as two commercial reference materials silicone Elastosil and VHB foil were evaluated

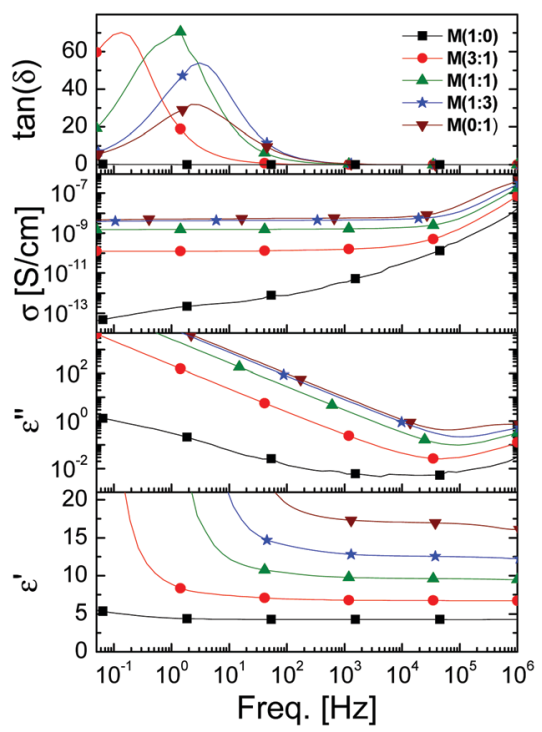

Fig. 6 Dielectric properties as a function of frequency of $M(x: y)$. regarding their actuation strain at different electric fields using circular actuators having $8 \mathrm{~mm}$ diameter carbon black electrodes. A biaxial prestrain of $28.6 \%$ was applied to all samples to avoid wrinkling of the electrode during actuation, except for VHB where $300 \%$ biaxial prestrain was used. The changes in the electrode diameter were measured while the voltage was increased (see the Experimental section). Fig. 7 shows the lateral actuation strain as a function of electric field for different materials $\mathbf{M}(\boldsymbol{x}: \boldsymbol{y})$. The actuation as a function of electric field and the stress-strain curves of Elastosil, VHB, and $\mathbf{M}(\boldsymbol{x}: \boldsymbol{y})$ are given in the ESI. $\dagger$

Table 5 summarizes the dielectric permittivity, $Y_{30 \%}$, electromechanical sensitivity $\varepsilon^{\prime} / Y_{10 \%}$, the actuation at different electric fields, some dielectric characteristics of materials $\mathbf{M}(\boldsymbol{x}: \boldsymbol{y})$ as well as of two commercial materials. For all materials two independent actuation tests were conducted except for $\mathbf{M}(3: \mathbf{1})$ for which only one actuator was measured. All materials, except for VHB actuated at electric fields lower than $20 \mathrm{~V} \mathrm{\mu m}^{-1}$ whereby $\mathbf{M}(0: 1)$ showed the largest actuation of $21 \%$ at the lowest field of $11.9 \mathrm{~V} \mathrm{\mu m}^{-1}$. Despite the slight increase in the elastic moduli at higher nitrile contents in $\mathbf{M}(\boldsymbol{x}: \boldsymbol{y})$, a rise of the actuation strain at a certain voltage with the increasing content of polar groups

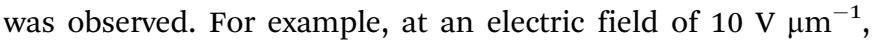
$\mathbf{M}(\mathbf{1}: 0)$ exhibits $2.7 \%$ strain, $\mathbf{M}(\mathbf{1}: 1)$ gave $6.4 \%$ strain, and $\mathbf{M}(\mathbf{0}: 1)$ reached a maximum actuation strain of $14.1 \%$. The reason behind the low electric field required for actuating $\mathbf{M}(\boldsymbol{x}: \boldsymbol{y})$ is the strong increase in permittivity for these materials, and therefore an increase in electromechanical sensitivity. It is generally accepted that the actuation strain increases with increasing $\varepsilon^{\prime} / Y$ values. Despite the lower elastic modulus of the two commercial materials, the silicone Elastosil and the acrylic VHB foil as compared to $\mathbf{M}(\boldsymbol{x}: \boldsymbol{y})$, their actuation strain is rather low. This is caused by low permittivity, and therefore low $\varepsilon^{\prime} / Y$ values. Novel, chemically modified silicone based materials reported in the literature also show reduced driving voltages at increased $\varepsilon^{\prime} / Y$ values which are mainly caused by a moderate increase of permittivity and a decrease of elastic modulus. ${ }^{17-19,31-33}$ This decrease with the increasing content of polar groups in the material is somewhat surprising as one would normally expect an

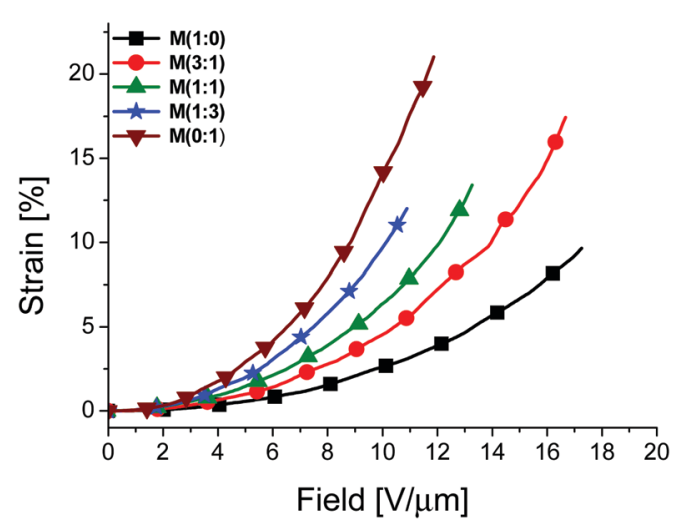

Fig. 7 Lateral actuation strain as a function of electric field for materials $M(x: y)$ which were $28.6 \%$ biaxially prestrained. The curves were averaged from two independent tests, except for $M(3: 1)$ for which only one measurement was conducted. 
Table 5 Permittivity $\left(\varepsilon^{\prime}\right)$ at $1 \mathrm{kHz}$, elastic modulus at 30\% strain $Y_{30 \%}$, lateral actuation strain at certain electric fields, the dielectric breakdown of actuators, the dielectric breakdown field $E_{\mathrm{b}}$ obtained by using a setup similar to that of Kollosche et al., ${ }^{35}$ the Weibull shape parameter $\beta$, the Weibull scale parameter $\eta$, and $R^{2}$ of the linear fit for $\mathrm{M}(\boldsymbol{x}: \boldsymbol{y})$

\begin{tabular}{|c|c|c|c|c|c|c|c|c|c|c|c|c|}
\hline Material & $\mathrm{CN}$ groups [mol\%] & $\varepsilon^{\prime}$ @ $1 \mathrm{kHz}$ & $Y_{10 \%}\left[\mathrm{~N} \mathrm{~mm}^{-2}\right]$ & $\varepsilon^{\prime} / Y_{10 \%}$ & $s^{a}[\%]$ & ${ }^{b} E\left[\mathrm{~V}_{\left.\mu \mathrm{m}^{-1}\right]}\right.$ & $s_{\max }[\%]$ & $E_{\mathrm{b}, \text { act }}\left[\mathrm{V} \mu \mathrm{m}^{-1}\right]$ & $E_{\mathrm{b}}{ }^{c}\left[\mathrm{~V} \mu \mathrm{m}^{-1}\right]$ & $\beta$ & $\eta$ & $R^{2}$ \\
\hline$M(3: 1)$ & 25 & 6.8 & 0.096 & 68 & 4.6 & 13.8 & 17.5 & 18 & $27.0 \pm 2.5$ & 12.0 & 28.1 & 0.96 \\
\hline$M(1: 1)$ & 50 & 9.8 & 0.097 & 81.7 & 6.4 & 11.9 & 13.5 & 13 & $21.2 \pm 2.5$ & 9.4 & 22.3 & 0.94 \\
\hline$M(0: 1)$ & 100 & 17.4 & 0.155 & 116 & 14.1 & 8.7 & 21.0 & 12 & $15.6 \pm 1.9$ & 8.6 & 16.4 & 0.96 \\
\hline Elastosil & - & 3 & 0.09 & 33.3 & 0.7 & - & 6 & 25 & - & - & - & - \\
\hline VHB & - & 4.4 & 0.2 & 22 & 0 & 68 & - & - & - & - & - & - \\
\hline E-DR19 & $7.1 \%^{d}$ & 4.64 & $0.325^{e}$ & 14.1 & $<1.5 \%$ & 57.8 & 17.6 & 68.2 & - & - & - & - \\
\hline E-DR19 & $10.3 \%^{d}$ & 4.82 & $0.367^{e}$ & 13.1 & $<1.5 \%$ & 54.2 & 11.7 & 56.8 & - & - & - & - \\
\hline E-DR19 & $13.3 \%^{d}$ & 4.88 & $0.725^{e}$ & 6.7 & $1.5 \%$ & 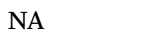 & 21. & .9 & - & - & - & - \\
\hline
\end{tabular}

${ }^{a}$ Lateral actuation strain at $10 \mathrm{~V} \mu \mathrm{m}^{-1} .{ }^{b}$ Electric field used to induce $9.7 \%$ lateral actuation strain. ${ }^{c} E_{\mathrm{b}}$ was determined by placing the dielectric film between electrodes of $1 \mathrm{~mm}^{2}$ area. ${ }^{d}$ wt\% of DR19 in materials is given. ${ }^{e}$ The elastic moduli at small strains. ${ }^{34}$

increase in stiffness caused by the dipolar interactions. ${ }^{29}$ The reason for this unexpected observation is a reduced crosslinking degree caused by the polar groups which resulted in a softer material.

A recent comprehensive overview regarding the performance of different polar silicone materials by Madsen et al. is available. ${ }^{4}$ Here, we compare our results with materials reported by Zhang et al. which behave similar to ours and where actuation tests were performed. Their silicone elastomers were modified with Disperse Red $19 .{ }^{34}$ An increase in permittivity from 2.72 to 4.88 and an increase in elastic modulus from $268 \mathrm{kPa}$ to $725 \mathrm{kPa}$ with the increasing content of DR19 were observed (Table 5). The $\varepsilon^{\prime} / Y$ values increase with the content of DR19 up to a concentration of $10.3 \mathrm{wt} \%$ when a decrease of the $\varepsilon^{\prime} / Y$ value was observed. Above this concentration, the actuation performance deteriorated. In our materials, no such saturation was observed, e.g. the $\varepsilon^{\prime} / Y$ values gradually increase with the nitrile content which is reflected in a linear increase in the actuation strain. In addition, our materials show significantly higher $\varepsilon^{\prime} / Y$ values. An actuation strain as high as $21 \%$ at an electric field as low as $11.9 \mathrm{~V} \mathrm{\mu m}^{-1}$ was achieved, while the DR19 modified materials reach a maximum actuation strain of $17 \%$ at significantly higher electric fields of about $68.2 \mathrm{~V} \mu \mathrm{m}^{-1}$.

The dielectric breakdown field $\left(E_{\mathrm{b}}\right)$ of the films was also measured. As expected, the higher the dipole content in $\mathbf{M}(\boldsymbol{x}: \boldsymbol{y})$, the lower the dielectric breakdown. This trend was also observed in actuator tests, except that the $E_{\mathrm{b}}$ values in actuators are slightly lower given the fact that the electrode area was significantly larger $\left(\sim 50 \mathrm{~mm}^{2}\right)$, and therefore the probability of failure increased.

The influence the $\mathrm{CN}$ content in the materials $\mathbf{M}(\boldsymbol{x}: \boldsymbol{y})$ has on the dielectric breakdown field was further investigated using Weibull statistics using eqn (1)

$$
\ln \left[\ln \frac{1}{(1-F)}\right]=\beta \ln \left(E_{\mathrm{b}}-\gamma\right)-\beta \ln (\eta)
$$

where $F$ represents the Weibull distribution function, ${ }^{36} \beta$ and $\eta$ are Weibull parameters, $\gamma$ is the Weibull location parameter,

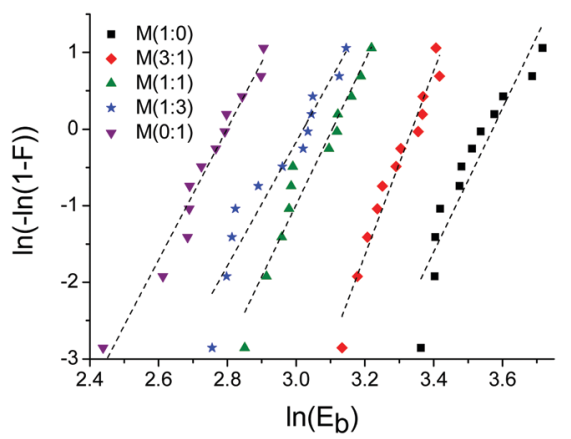

Fig. 8 Weibull plot for different materials $M(x: y)$. The dashed lines represent the linear fit to the data.

and $E_{\mathrm{b}}$ the dielectric breakdown in the samples. Fig. 8 shows the Weibull fits for different materials.

Since the points are in good agreement with the linear fit, the Weibull location parameter $\gamma$ was neglected. Sample $\mathbf{M}(\mathbf{1}: \mathbf{0})$ is an exception $\left(R^{2}=0.87\right)$ because the points might fit better to a concave curve which might suggest that $\gamma$ has to be considered. The corresponding calculations of sample $\mathbf{M}(\mathbf{1}: \mathbf{0})$ for which $\gamma \neq 0$, can be found in the ESI. $\dagger$ The Weibull shape parameter $\beta$ corresponds to the slope of the linear fit and gives information about the shape of the distribution. The $\beta$ values lie in a narrow range between 8.1 and 12.0 indicating a similar shape of the distribution for all materials. The Weibull scale parameter $\eta$ was determined for $F=0.632$, were $\eta$ is equal to $E_{\mathrm{b}}$ and can therefore easily be calculated using eqn (2):

$$
\eta=\mathrm{e}^{\ln \left[\ln \frac{1}{1-F}\right]-b_{\mathrm{i}}}
$$

using the Weibull plot intercept $b_{\mathrm{i}}$. The determined Weibull parameters $\beta$ and $\eta$, the average dielectric breakdown field as well as the $R^{2}$ of the linear fit are shown in Table 5. As already suggested by the dielectric measurements, a decrease in $\eta$ with the increasing content of $\mathrm{CN}$ can be seen. The breakdown field at a cumulative probability of $63.2 \%$ decreases from 
35.6 $\mathrm{V} \mu \mathrm{m}^{-1}$ for $\mathbf{M}(\mathbf{1}: \mathbf{0})$ to $22.3 \mathrm{~V} \mu \mathrm{m}^{-1}$ for $\mathbf{M}(\mathbf{1}: \mathbf{1})$ to $16.4 \mathrm{~V} \mu \mathrm{m}^{-1}$ for $\mathbf{M}(\mathbf{0}: \mathbf{1})$. Despite the reduction of the breakdown field and the increase of the elastic moduli with the $\mathrm{CN}$ content, the maximum actuation strain of $21 \%$ at $12 \mathrm{~V} \mathrm{\mu m}^{-1}$ is about two times higher as compared to the actuation strain of 9.7 at an almost double electric field of $23 \mathrm{~V} \mathrm{~m}^{-1}$ for material $\mathbf{M}(\mathbf{0}: 1)$ which has no $\mathrm{CN}$ groups. The large actuation strain of $\mathbf{M}(\mathbf{0}: \mathbf{1})$ is highly attractive since this opens the access to actuators operable below $100 \mathrm{~V}$ with dielectric films of $10 \mu \mathrm{m}$. Work in this direction is on the way.

\section{Conclusions}

Polysiloxane elastomers with different contents of polar nitrile groups and about the same degree of chemical cross-linking were prepared in a one-step process starting from a high molecular weight poly(methylvinylsiloxane) by using thiol-ene photo-addition of butanethiol (1), 3-mercaptopropionitrile (2) or a mixture thereof, and the 2,2'-(ethylenedioxy)diethanethiol (3) cross-linker to the vinyl groups. The resulting elastomers were characterized by IR, tensile tests, DMA, TGA, DSC, impedance spectroscopy as well as by electromechanical tests using circular actuators. An increase in stiffness, strain at break, and tensile stress with the increasing content of polar nitrile groups in the elastomers with a maximum elongation at break of $320 \%$ for an elastomer that contained the maximum amount of nitrile groups was observed. DMA shows an increase in stiffness with the increasing content of polar groups and low viscoelastic losses at low frequency. The mechanical losses at high frequencies increase with the increasing content of nitrile groups but stay below 0.4. TGA shows that the elastomers are stable up to $300{ }^{\circ} \mathrm{C}$, while according to DSC these elastomers have a $T_{\mathrm{g}}$ well below room temperature. Impedance spectroscopy investigations show that the permittivity can be fine-tuned from $\varepsilon^{\prime}=4.3$ for the elastomer modified only with butanethiol to the maximum value of $\varepsilon^{\prime}=17.4$ for the elastomer that has the maximum content of nitrile groups. All other elastomers lie within this range. Electromechanical tests showed that the actuation field is significantly reduced for the nitrile modified elastomers. For example, the actuation strain at $10 \mathrm{~V} \mathrm{\mu m}^{-1}$ of the material modified with 1 was $2.7 \%$, increased to $6.4 \%$ for an elastomer modified with a 1:1 mixture of thiols 1 and 2 , and reached a value of $14.1 \%$ for the elastomer containing the maximum amount of nitrile groups. The actuation strain of $21 \%$ at about

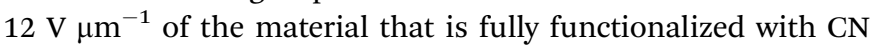
groups is highly attractive since this opens the possibility of constructing actuators operable below $100 \mathrm{~V}$ with dielectric films whose thickness is below $10 \mu \mathrm{m}$. Low driving voltage actuators are highly attractive since the electronics required to drive them are simpler and cheaper. Work in this direction is on the way.

\section{Acknowledgements}

We gratefully acknowledge the Swiss National Science Foundation (SNF132101, Swiss-Romanian Cooperation Program, grant no. IZERZO_142215/1) and Swiss Federal Laboratories for Materials
Science and Technology (Empa, Dübendorf) for financial support. We also acknowledge B. Fischer for DSC and TGA measurements, C. Walder and T. Künniger for their help with the DMA measurements, and Dr G. Kovacs for providing us with the infrastructure for the actuator measurements (all Empa). Many thanks to Prof. Z. M. Dang from Tsinghua University, Beijing, for providing us with the data of his materials.

\section{Notes and references}

1 R. Pelrine, R. Kornbluh, Q. Pei and J. Joseph, Science, 2000, 287, 836-839.

2 W. Hu, S. N. Zhang, X. Niu, C. Liu and Q. Pei, J. Mater. Chem. C, 2014, 2, 1658-1666.

3 J. E. Q. Quinsaat, M. Alexandru, F. A. Nüesch, H. Hofmann, A. Borgschulte and D. M. Opris, J. Mater. Chem. A, 2015, 3, 14675-14685.

4 F. B. Madsen, A. E. Daugaard, S. Hvilsted and A. L. Skov, Macromol. Rapid Commun., 2016, 37, 378-413.

5 A. O'Halloran, F. O'Malley and P. McHugh, J. Appl. Phys., 2008, 104, 071101.

6 R. E. Perline, R. D. Kornbluh and J. P. Joseph, Sens. Actuators, A, 1998, 64, 77-85.

7 D. M. Opris, S. Dünki, C. Racles, A. Bele and M. Cazacu, PCT Int. Appl., WO 2015135086 A1 20150917, 2015.

8 M. Stepp, F. Achenbach and A. Koellnberger, WO 2015/ $121261 \mathrm{Al}, 2015$.

9 D. M. Opris and S. Dünki, Chimia, 2015, 69, 549.

10 D. M. Opris, J. E. Q. Quinsaat, S. Dünki, Y. S. Ko, M. Alexandru, C. Racles and F. A. Nüesch, Proc. SPIE, 2015, 94300A.

11 P. Brochu and Q. Pei, Macromol. Rapid Commun., 2010, 31, 10.

12 R. Shankar, T. K. Ghosh and R. J. Spontak, Adv. Mater., 2007, 19, 2218-2223.

13 L. Maffli, S. Rosset, M. Ghilardi, F. Carpi and H. Shea, Adv. Funct. Mater., 2015, 25, 1656.

14 L. J. Romasanta, M. A. Lopez-Manchado and R. Verdejo, Prog. Polym. Sci., 2015, 51, 188.

15 F. B. Madsen, L. Yu, A. E. Daugaard, S. Hvilsted and A. L. Skov, RSC Adv., 2015, 5, 10254.

16 F. B. Madsen, L. Yu, A. E. Daugaard, S. Hvilsted and A. L. Skov, Polymer, 2014, 55, 6212.

17 A. Risse, B. Kussmaul, H. Krüger and G. Kofod, Adv. Funct. Mater., 2012, 22, 3958-3962.

18 B. Kussmaul, S. Risse, G. Kofod, R. Wache, M. Wegener, D. N. McCarthy, H. Krüger and R. Gergard, Adv. Funct. Mater., 2011, 21, 4589-4594.

19 H. Böse, D. Uhl and R. Rabindranath, Proc. SPIE, 2012, 8340, 83402E.

20 A. H. A. Razak, P. Szabo and A. L. Skov, RSC Adv., 2015, 5, 53054.

21 S. J. Dünki, M. Tress, F. Kremer, S. Y. Ko, F. A. Nüesch, C.-D. Varganici, C. Racles and D. M. Opris, RSC Adv., 2015, 5, 50054-50062.

22 C. Racles, M. Alexandru, A. Bele, V. E. Musteata, M. Cazacu and D. M. Opris, RSC Adv., 2014, 4, 37620-37628. 
23 S. J. Dünki, Y. S. Ko, F. A. Nüesch and D. M. Opris, Adv. Funct. Mater., 2015, 25, 2467-2475.

24 M. Mooney, J. Appl. Phys., 1940, 11, 582.

25 R. S. Rivlin, Philos. Trans. R. Soc., A, 1948, 241, 379.

26 R. E. Gerber, C. Hasbun, L. G. Dubenko, M. F. King and D. E. Bierer, Org. Synth., 2000, 77, 86.

27 Y. Zhang, Y. Li and W. Liu, Adv. Funct. Mater., 2015, 25, 471-480.

28 T. Bai, P. Zhang, Y. Han, Y. Liu, W. Liu, X. Zhao and W. Lu, Soft Matter, 2011, 7, 2825-2831.

29 S. Thomas, C. H. Chan, L. A. Pothen, K. R. Pajisha and

H. L. Maria, Natural Rubber Materials, RSC Publishing, 2014.
30 F. Kremer and A. Schönhals, Boadband Dielectric Spectroscopy, Springer-Verlag, Berlin Heidelberg, 2003.

31 B. Kussmaul, S. Risse, M. Wegener, G. Kofod and H. Krüger, Smart Mater. Struct., 2012, 21, 064005.

32 S. Risse, B. Kussmaul, H. Krüger and G. Kofod, RSC Adv., 2012, 2, 9029.

33 M. Dascalu, S. J. Dünki, J.-E. Q. Quinsaat, Y. S. Ko, F. A. Nüesch and D. M. Opris, RSC Adv., 2015, 5, 104516.

34 L. Zhang, D. Wang, P. Hu, J.-W. Zha, F. Yous, S.-T. Li and Z. M. Dang, J. Mater. Chem. C, 2015, 3, 4883-4889.

35 M. Kollosche and G. Kofod, Appl. Phys. Lett., 2010, 96, 12-15. 36 IEC 61649:2008. 